\title{
A characterization of cis- and trans-heritability of RNA-Seq-based gene expression
}

\author{
Klaasjan G. Ouwens $\mathbb{D}^{1} \cdot$ Rick Jansen $\mathbb{D}^{2} \cdot$ Michel G. Nivard $\mathbb{D}^{1} \cdot$ Jenny van Dongen ${ }^{1} \cdot$ Maia J. Frieser ${ }^{3,4}$.

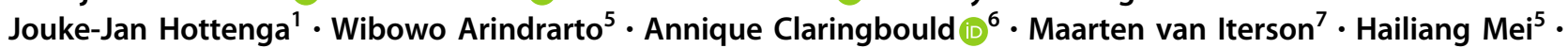 \\ Lude Franke $\mathbb{1}^{6} \cdot$ Bastiaan T. Heijmans ${ }^{7} \cdot$ Peter A. C. 't Hoen $\mathbb{D}^{8,9}$. Joyce van Meurs ${ }^{10}$. Andrew I. Brooks ${ }^{11}$. \\ BIOS Consortium • Brenda W. J. H. Penninx ${ }^{2} \cdot$ Dorret I. Boomsma ${ }^{1}{ }^{1}$
}

Received: 6 November 2018 / Revised: 21 July 2019 / Accepted: 12 August 2019 / Published online: 26 September 2019

(c) The Author(s), under exclusive licence to European Society of Human Genetics 2019

\begin{abstract}
Insights into individual differences in gene expression and its heritability $\left(h^{2}\right)$ can help in understanding pathways from DNA to phenotype. We estimated the heritability of gene expression of 52,844 genes measured in whole blood in the largest twin RNA-Seq sample to date (1497 individuals including 459 monozygotic twin pairs and 150 dizygotic twin pairs) from classical twin modeling and identity-by-state-based approaches. We estimated for each gene $h_{\text {total }}^{2}$, composed of cisheritability ( $h_{\text {cis }}^{2}$, the variance explained by single nucleotide polymorphisms in the cis-window of the gene), and trans-

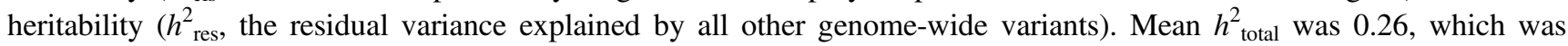
significantly higher than heritability estimates earlier found in a microarray-based study using largely overlapping $(>60 \%)$ RNA samples (mean $h^{2}=0.14, p=6.15 \times 10^{-258}$ ). Mean $h_{\text {cis }}^{2}$ was 0.06 and strongly correlated with beta of the top cis expression quantitative loci (eQTL, $\rho=0.76, p<10^{-308}$ ) and with estimates from earlier RNA-Seq-based studies. Mean $h^{2}$ res was 0.20 and correlated with the beta of the corresponding trans-eQTL $\left(\rho=0.04, p<1.89 \times 10^{-3}\right)$ and was significantly higher for genes involved in cytokine-cytokine interactions $\left(p=4.22 \times 10^{-15}\right)$, many other immune system pathways, and genes identified in genome-wide association studies for various traits including behavioral disorders and cancer. This study provides a thorough characterization of $c i s$ - and trans- $h^{2}$ estimates of gene expression, which is of value for interpretation of GWAS and gene expression studies.
\end{abstract}

\section{Introduction}

Individual differences in RNA expression may result from variation in environmental exposures, stochastic variation, age, sex and genotype differences [1] and thereby may also be involved in the widely observed contribution of DNA, age and sex to the etiology of complex diseases [2-5]. Quantifying human transcriptomic heritability $\left(h^{2}\right)$ is of interest for transcriptomic and genomic studies. For

Members of the BIOS Consortium are listed at the end of the paper.

Supplementary information The online version of this article (https:// doi.org/10.1038/s41431-019-0511-5) contains supplementary material, which is available to authorized users.

Klaasjan G. Ouwens

klaasjan.ouwens@vu.nl

Extended author information available on the last page of the article example, one possible reason for the association of gene expression with a certain phenotype is that DNA variants influence the amount of gene expression through expression quantitative trait loci (eQTLs). Gene expression can have substantial $h^{2}$ not explained by eQTLs [6], and therefore the $h^{2}$ of gene expression and corresponding eQTL findings can be considered complementary to transcriptomic and genomic studies.

The $h^{2}$ of whole blood gene expression has been established previously for genome-wide transcriptomic data generated by microarray technology and by RNA-Seq. For array technology, Wright et al. [7] found a mean gene expression $h^{2}$ of $0.10(\mathrm{SD}=0.14, N=2752,18,392$ genes $)$ from modeling data assessed in mono- and dizygotic twin pairs. Local identity-by-descent (IBD) analyses, which provide an estimate of variance of gene expression explained by genetic relatedness, resulted in a mean local $h^{2}$ of 0.03 , explaining $23 \%$ of the total heritability. A population-based study $(N=2765)$ by Lloyd-Jones et al. [6] 
found mean estimates of $h^{2}$ of microarray-based gene expression of 0.19 with a local $h^{2}$ of 0.06 , resulting in a mean proportion of genetic variance explained by all eQTLs of $31 \%$. Viñuela et al. [8] estimated a mean of bloodderived $h^{2}$ of 0.23 in a twin-based sample $(N=855)$ for RNA-Seq-based data.

Here, we analyzed RNA-Seq (Illumina Hiseq2000) data from $459 \mathrm{MZ}$ twin pairs, $150 \mathrm{DZ}$ twin pairs, 24 relatives of twin pairs, and 255 unrelated participants, leading to a total dataset with genotype and expression data of 1497 adult participants (998 females) from the Netherlands Twin Register [9-11] (Supplementary Table 1). Our RNA-Seq dataset contained a large $(>60 \%)$ sample overlap with the microarray-based dataset from Wright et al. [7], allowing a reliable comparison between microarray and RNA-Seqbased $h^{2}$ estimates. We first estimated twin-based $h^{2}$ of gene expression by making use of the classical twin design [12]. Next, we simultaneously estimated the variance explained by a genetic relationship matrix (GRM)-containing SNPs in a 250 kilobase $(\mathrm{kb})$ cis-window of the gene $\left(h^{2}\right.$ is $)$, and the variance explained by a second GRM including only closely related individuals $\left(h_{\text {res }}^{2}\right)$ using GCTA [13]. Together, $h_{\text {cis }}^{2}$ and $h_{\text {res }}^{2}$ constitute the total heritability $\left(h_{\text {total }}^{2}\right)$. We performed cis- and trans-eQTL analyses of the same samples and compared $h^{2}$ and eQTL findings to test for consistency.

\section{Methods}

\section{Participants}

RNA samples were obtained from 1497 participants from the Netherlands Twin Registry (NTR) and included 459 complete MZ twin pairs and 150 complete DZ twin pairs, 24 relatives of twin pairs, and 255 unrelated participants. NTR is a longitudinal cohort study of twins and their families $[10,14]$. The age of the participants ranged from 17.6 to 79.6 years old (mean $=36.7, \mathrm{SD}=14.0$ ), $67 \%$ of the sample was female. The data used for this study largely overlap (60\%) with those used in an earlier study [7]. See Supplementary Table 1 for a description of the samples.

\section{RNA extraction and sequencing}

Venous samples were drawn in the morning after an overnight fast. Heparinized whole blood samples were transferred within $20 \mathrm{~min}$ of sampling into PAXgene Blood RNA tubes (Qiagen, Valencia, CA, USA) and stored at $-20^{\circ} \mathrm{C}$. Total RNA from whole blood was depleted of globin transcripts using the Ambion GLOBINclear kit and subsequently processed for sequencing using the Illumina TruSeq version 2 library preparation kit. Paired-end sequencing of $2 \times 50$-bp reads was performed using the
Illumina HiSeq 2000 platform, pooling ten samples per lane and aiming for $>15$ million read pairs per sample. Adapters were identified and clipped, and low-quality read ends were trimmed (min length 25, min quality 20 Read alignment was performed using STAR 2.3.0e against the Genome of the Netherlands (GoNL) reference panel [15]. Expression was using Ensembl v.71 annotation (which corresponds to GENCODE v.16). Overlapping exons (on either of the two strands) were merged into meta-exons, and expression was quantified for the whole meta-exon, resulting in base counts per exon or meta-exon. Gene expression, as base count per gene, was calculated as the sum of the expression values for all exons of each gene (excluding meta-exons). This pipeline is explained in detail in Zhernakova et al. [16].

Gene expression values per gene were ranked and mapped to a normal distribution with mean 0 and SD 1, after which values were corrected for sex, age, and cell counts: monocyte, lymphocyte, eosinophil, basophil, neutrophil and red blood cell counts, and 27 measurement batches. We then performed a principal-component analysis, and values were corrected on only the first principal component, which was not heritable based on comparison of goodness of fit between different twin-based structural equation models $(p=0.74)$. This PC explained $16.5 \%$ of the variance in the data (see Supplementary Figs. 1 and 2). Finally, expression values were centered and subsequently scaled by dividing these values by their respective standard deviations. Analyses of both classical twin modeling-based and identity-by-state (IBS)-based $h^{2}$ were based on this final dataset.

\section{Genotype data}

Within the NTR, genotype information is available for 15,111 individuals for four different genotyping arrays (Affymetrix $6.0(N=11,781)$, Affymetrix Perlegen 5.0 $(N=1265)$, Illumina $660(N=1439)$ and Illumina Omni Express $1 \mathrm{M}(N=257)$, as well as sequence data from the Netherlands reference genome project GONL (BGI full sequence at $12 \times(N=368$; [17]). Samples were removed if they had a genotype call rate below $90 \%$, heterozygosity fell outside the range of -0.075 to 0.075 , gender and IBS status mismatch occurred, or if the Mendelian error rate was larger than 5 standard deviations from the mean of all samples and for samples measured on Affymetrix 6.0 when the contrast quality control value was smaller than 0.40 . Quality control of the SNPs was done for each platform separately, with SNPs being removed when they could only be aligned to the forward strand of the reference panel, the allele frequencies differed more than $10 \%$ with the reference allele, minor allele frequency (MAF) was below 0.005, Hardy-Weinberg equilibrium (HWE) test $p<10^{-12}$, and a genotype call rate of $<0.95$. The data of the different 
genotyping methods, except GONL sequence individuals, were subsequently merged into a single dataset. The missing SNP genotypes between each platform were imputed to the GONL reference data. Filtering of the imputed dataset included the removal of SNPs which were significantly associated with a single genotyping platform, if the allele frequency difference differed more than $10 \%$ with the GONL reference set, HWE $p<10^{-5}$, Mendelian error rate $>$ mean $+5 \mathrm{SD}$ and if the imputation quality (R2) was below 0.90. After filtering the GONL samples were readded to a cross-platform imputed dataset that includes $1,261,818$ SNPs. We did not perform additional genotype quality control after subsetting the individuals with RNAseq.

\section{Statistical methods}

We employed two methods for estimating total heritability, a classical twin modeling-based approach, and an IBSbased approach. The classical twin modeling approach requires information on relatedness between subjects, while the IBS-based approach quantifies the genetic relatedness between subjects based on their genome-wide genetic data. For both methods, only autosomal genes were considered.

\section{Classical twin modeling}

Based on the resemblance of MZ and DZ twin pairs, the variance of the expression of each gene can be decomposed into additive genetic, common (or shared) environmental and unique environmental variance. Classical twin-based modeling was done in the structural equation modeling (SEM) R-package OpenMx [18, 19]. Models were fitted to decompose the variance of gene expression due to additive genetic (A), shared environmental (C), and unique environmental (E) effects. Additive genetic effects combine all the effects of genetic variants influencing gene expression. Shared environmental variance represents the proportion of variance explained by effects are shared by both members of a twin pair. Unique environmental variance results from environmental effects that are not shared by twins. We used a standard ACE model assuming dizygotic twins have an average IBD sharing of 0.5 across the genome, and monozygotic twins share an IBD of 1 . Parameters were estimated by maximum likelihood (ML). We restricted the estimates to be positive. The results of these analyses provide the ML estimates of variance components. The comparison of ACE and CE models gave an estimate for the significance of the A-component.

\section{IBS-based analysis}

Techniques to quantify genetic similarity of 'unrelated' individuals who are genotyped for a large number of
SNPs across the entire genome have been developed to estimate $h^{2}$ due to SNPs. In software packages such as GCTA [13] the relatedness among individuals based on measured SNPs can be combined with known genetic relatedness in relatives in a two-variance component linear mixed model [20], in which simultaneous estimation of SNP heritability and total $h^{2}$ is feasible. We used NTR genotype information for the 1497 individuals for whom both expression- and genotype data were available. A GRM was created for each cis-window of a gene, defined by the coordinates of a gene with $250 \mathrm{~kb}$ flanking area on each side. This GRM is referred to as the cis-GRM and represents the variance explained by all measured SNPs (and SNPs tagged by these measured SNPs) in a ciswindow around the gene of $250 \mathrm{~kb}$. Variance explained by this $c i s-G R M$ is referred to as $c i s-h^{2}\left(h^{2}{ }_{\text {cis }}\right)$. The mean number of SNPs in cis-GRMs was $223.2(\mathrm{SD}=119.9)$ (see Supplementary Fig. 3). A second GRM including closely-related individuals (that is, a genetic correlation > 0.05) was created for the autosomes. This GRM is referred to as the residual GRM. This GRM had all off-diagonal elements below 0.05 set to 0 , to remove distant relatedness from the matrix. Variance explained by this residual GRM is referred to as residual $h^{2}\left(h^{2}{ }_{\text {res }}\right)$. The sum of $h_{\text {cis }}^{2}$ and $h_{\text {res }}^{2}$ is $h_{\text {total }}^{2}\left(h_{\text {total }}^{2}=h_{\text {cis }}^{2}+h^{2}{ }_{\text {res }}\right)$. As the cis-GRM is based on a limited number of SNPs there is substantial power to detect the genetic effects in cis [21], the second GRM will absorb all genetic variance not explained by the SNPs in the cis-window, or in high LD with SNPs in the cis-window. Note that due to the presence of a large number of related individuals this GWAS will capture genetic variance tagged by substantial IBD sharing and thus the sum of the two effects will be approximately equal to the heritability estimated in a twin study.

$\operatorname{cov}(\text { expression })_{n * n}=\mathrm{GRM}_{n * n}^{\mathrm{IBS}} \Theta \sigma_{c i s-\mathrm{SNPs}}^{2}+\mathrm{GRM}_{n * n}^{\mathrm{IBS}>0.05} \Theta \sigma_{\mathrm{SNPs}}^{2}+I_{n * n} \Theta \sigma_{\mathrm{e}}^{2}$ A total of 52,844 genes were analyzed, and subsequently filtered for being protein coding, having read counts above zero in at least $85 \%$ samples in each zygosity group (e.g. expressed in in at least $780 \mathrm{MZ}$ twins and $255 \mathrm{DZ}$ twins), a median expression count above 10, and more than 20 SNPs in the cis-window, resulting in an analysis of 11,353 genes (Supplementary Table 2).

\section{Annotation and enrichment}

We employed multiple annotation steps to interpret $h^{2}$ estimates. We tested whether $h^{2}$ was correlated with gene expression level, gene length, GC content or several lossof-function scores obtained from Lek et al. [22] using linear regression. Gene locations and lengths were downloaded on 2017-12-28 using the Biomart community portal [23] using data from Ensembl [24]. We investigated 
genes for which the expression correlates highly $(>0.8)$ with other genes.

In addition, we tested whether heritable genes are overrepresented in the canonical gene pathways from the molecular signature database (MSIGDB):: KEGG, REACTOME, BIOCARTA pathways downloaded from http:// software.broadinstitute.org/gsea/downloads.jsp (c2.cp.v6.1) and genes identified in GWAS for immune diseases, mental or behavioral disorders, cardiovascular diseases, or cancer (extracted from the GWAS catalog [25] (as of September 2018) with the search terms: 'immune system disease', 'mental or behavioral disorder', 'cardiovascular disease' or 'cancer', respectively). For each pathway/gene group, a Wilcoxon test was performed between the median heritability of the genes in the pathway and the median heritability of the genes outside the pathway. Comparisons were made with previous analyses of $h^{2}$ of gene expression from a recent whole blood-based RNA-Seq study by Battle et al. [26] and the GTEx project [27], which published heritability estimates for gene expression in adipose tissue (subcutaneous), tibial artery, heart (left ventricle), lung, muscle (skeletal), tibial nerve, skin (sun-exposed), thyroid, and whole blood.

\section{eQTL analysis}

The 1497 gene-level RNA samples with the same preprocessing as described above which were also used for heritability analysis, were also used for cis- and trans-eQTL analysis. For this analysis, the same cross-platform imputed dataset that includes $1,261,818$ SNPs as described above, was filtered at MAF $>0.01$ and $\mathrm{HWE}<1 \times 10^{-3}$, resulting in $1,239,670$ SNPs.

For $c i s$-eQTL analysis all associations between DNA variants and genes at distance $<250 \mathrm{~kb}$ were computed, for trans-eQTL analysis all SNP - gene pairs at distance $>250 \mathrm{~kb}$. eQTL effects were detected with a mixed linear model approach using fastGWA a implemented in GCTA (https://cnsgenomics.com/software/gcta/\#fastGWA, https://www.biorxiv.org/content/10.1101/598110v1).

For fastGWA, first a GRM is built using the -make-grm option in GCTA. Then, a sparse GRM is built using the option -make-bK-sparse 0.05. fastGWA is then run using this sparse GRM, with expression level as the dependent variable and SNP genotype values as independent variable. Correction for multiple testing was done using FDR, for $c i s-$ and trans-eQTL analysis separately, resulting in a $P$-value threshold of $1 \times 10^{-5}$ for cis-eQTLs, and $1.5 \times 10^{-7}$ for trans-eQTLs. We are aware that FDR used like this may result in more false positives than $5 \%$, however, we are merely interested in the overlap between heritability and eQTL analysis and do not draw any conclusions on the amount of identified eQTLs.

\section{Simulation}

To gain insight in the performance of our models, we simulated twin-based phenotype data with prespecified heritabilities and tested whether our estimations were in accordance. We ran OpenMX and GCTA models using simulated phenotypes and real genotype data, with different values for variance components (see Supplementary Figs. 6 and 7).

\section{Results}

The gene-level whole blood RNA-Seq data from 1497 participants, including $459 \mathrm{MZ}$ twin pairs and $150 \mathrm{DZ}$ twin pairs, originally contained 52,844 genes (Supplementary Table 2). After filtering (see "Methods"), 11,409 protein coding genes that were expressed in at least $85 \%$ of the samples were left for analysis. In these genes, twin-based $h^{2}$ for each gene was estimated based on the classical twin design (see Methods). Next, IBS-based methods were applied to compute SNP heritability.

For the twin-based analyses, a genetic structural equation model [28] that included additive genetic, common, and unique environmental factors was fitted to the expression data from each gene. The mean $h^{2}$ (the standardized estimate of the contribution of additive genetic factors) was estimated at .20, the standardized mean contribution of shared environment at 0.05 , and the standardized mean unshared environment at 0.74 . There was a considerable spread in estimates, with estimates ranging between 0 and 1 , except for the common environment component which had a maximum of 0.51 (Fig. 1 and Table 1).

Since estimates for the contribution of common environment on average were low, we proceeded with an IBSbased approach that did not take into account common environment. Gene expression is controlled by both local (cis) eQTLs and non-local (trans) eQTLs. Therefore, when studying the genetic component of expression in terms of heritability, we also make a distinction between the genetic component that is close to the gene $\left(h_{\text {cis }}^{2}\right)$, and the genetic component that is not close to the gene $\left(h_{\text {res }}^{2}\right) . h_{\text {cis }}^{2}$ consisted of the variance explained by SNPs in a $250-\mathrm{kb}$ ciswindow of the gene and $\mathrm{h}^{2}$ res the variance explained by genome-wide close relatedness. From the IBS approach we obtained heritability estimates for 11,353 out of 11,409 genes. The correlation between IBS-based total $h^{2}\left(h_{\text {cis }}^{2}+h_{\text {res }}^{2}\right)$ and classical twin modeling-based $h^{2}$ was $0.98\left(p<10^{-308}\right.$, Supplementary Fig. 4). The IBS-based approach resulted in a mean $h_{\text {res }}^{2}$ of 0.20 and a mean $h_{\text {cis }}^{2}$ of .06 (Fig. 1 and Table 1), summing up to a $h_{\text {total }}^{2}$ of 0.26 . We found 721 genes to have a Bonferroni-corrected significant $h_{\text {cis }}^{2}\left(p<4.40 \times 10^{-6}\right)$, all of which had a $h_{\text {cis }}^{2}$ larger than 
A

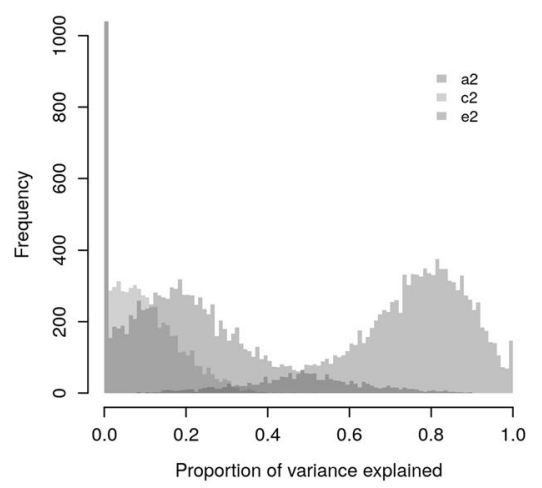

Fig. 1 Histograms of estimates of heritability of gene expression. a Classical twin modeling-based estimates of each gene. Based on the resemblance of MZ and DZ twin pairs, the variance of the expression of each gene was decomposed into additive genetic, common (or shared) environmental, and unique environmental variance. A standard ACE model was used, assuming dizygotic twins have an average IBD sharing of 0.5 across the genome, and monozygotic twins share an
B

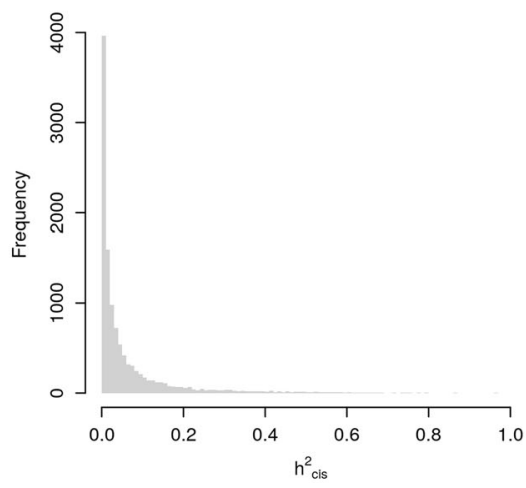

C

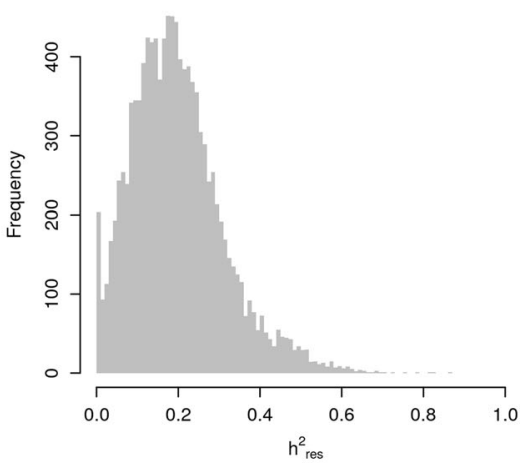

IBD of 1. Parameters were estimated by maximum likelihood (ML). b IBS-based $h_{\text {cis }}^{2}$ estimates of each gene, i.e. variance explained by a GRM created for each cis-window of a gene, defined by the coordinates of a gene with 250 kilobase $(\mathrm{kb})$ flanking area on each side. c IBS-based $h_{\text {res }}^{2}$ estimates of each gene, i.e. variance explained by a GRM including closely related individuals for the autosomes with all off-diagonal elements below 0.05 set to 0

Table 1 Estimates for classical twin modeling-based and IBS-based heritability of gene expression

\begin{tabular}{lllll}
\hline Variance component & Min & Median & Mean & Max \\
\hline $\mathrm{A}$ & 0 & 0.1793 & 0.2042 & 0.8988 \\
$\mathrm{C}$ & 0 & 0 & 0.0532 & 0.5125 \\
$\mathrm{E}$ & 0.0800 & 0.7702 & 0.7426 & 1 \\
$h^{2}$ cis & 0.0001 & 0.0212 & 0.0638 & 0.9666 \\
$h^{2}$ res & 0.0001 & 0.1848 & 0.1980 & 0.8655 \\
$h^{2}$ total & 0.0001 & 0.2338 & 0.2618 & 0.9874 \\
\hline
\end{tabular}

Genes were filtered for being protein coding, having less than $50 \mathrm{DZ}$ twins with zero expression counts, a median expression count above 10 , and more than 20 SNPs in the cis-window, resulting in a total of 11,353 genes tested. Classical twin-based modeling was done in the structural equation modeling (SEM) R-package OpenMx [18, 19]. Models were fitted to decompose the variance of gene expression due to additive genetic (A), shared environmental (C), and unique environmental (E) effects. $h_{\text {cis }}^{2}$ is the variance explained by measured SNPs in a cis-window around the gene of $250 \mathrm{~kb} . h_{\text {res }}^{2}$ is the variance explained by genome-wide closely related individuals

0.16 . We found 5636 genes to have a Bonferroni-corrected significant $h_{\text {res }}^{2}\left(p<4.40 \times 10^{-6}\right)$, all of which had a $h_{\text {res }}^{2}$ larger than 0.01 (Supplementary Table 3, Supplementary Fig. 5). The mean contribution of $h_{\text {cis }}^{2}$ to the total $h^{2}$ (mean $h_{\text {cis }}^{2} /\left(\right.$ mean $h_{\text {cis }}^{2}+$ mean $\left.h_{\text {res }}^{2}\right)$ ) was $20 \%$, with a range from 0 to $100 \%$. The correlation between $h_{\text {res }}^{2}$ and $h_{\text {cis }}^{2}$ was 0.06 $\left(p=6.36 \times 10^{-10}\right)$. We found good performance of our models, slightly underestimating local heritability (see Supplementary Figs. 6 and 7).

\section{Correlation of gene expression $h^{2}$ between tissues}

A whole blood-based RNA-Seq study by Battle et al. [26] published heritability estimates due to regulatory genetic variation $(N=922)$. Estimates of heritability due to local genetic variation from this study correlated 0.81 with $h_{\text {cis }}^{2}$ from our study $\left(p<10^{-308}\right.$, Spearman correlation, Supplementary Fig. 8). eQTL studies have shown that gene expression in different tissues is regulated by DNA by partially overlapping, and partially unique QTLs (https:// science.sciencemag.org/content/348/6235/648). To study the extent to which the total local genetic component of gene expression is similar between tissues, we looked at $h^{2}$ estimates of gene expression in The Genotype-Tissue Expression (GTEx). The GTEx consortium, $(N=422)$ [29] reported $h^{2}$ estimates for gene expression from RNA-Seq in adipose tissue (subcutaneous), tibial artery, heart (left ventricle), lung, muscle (skeletal), tibial nerve, skin (sunexposed), thyroid, and whole blood, estimating local $h^{2}$ both unique for a tissue (tissue specific) and heritability shared between tissues (tissue wide). Our estimates of $h_{\text {cis }}^{2}$ showed significant correlations with local $h^{2}$ in every measured tissue, in both tissue-wide $(\rho>0.12)$ and tissuespecific heritability $(\rho>0.03)$ estimates. The strongest correlation was found between $h_{\text {cis }}^{2}$ and tissue-wide heritability of whole blood-derived gene expression $(\rho=0.24$, $p=1.99 \times 10^{-144}$, Fig. 2, Supplementary Table 4). Heritability estimates reported in the GTEx study correlated $<.44$ between tissues in tissue-wide estimates and $<0.19$ in tissue-specific estimates.

Correcting $h_{\text {cis }}^{2}$ for median read count only showed marginal effects on correlations. There were 614 genes that were highly expressed in all tissues in GTEx (above the 8 th decile). These genes showed decreased $h_{\text {cis }}^{2}(p=5.58 \times$ $10^{-12}$, mean $=0.04$, median $\left.=0.02\right)$ and increased $h_{\text {res }}^{2}(p$ $=7.77 \times 10^{-14}$, mean $=0.23$, median $=0.22$ ) compared to the full set of tested genes. 
A

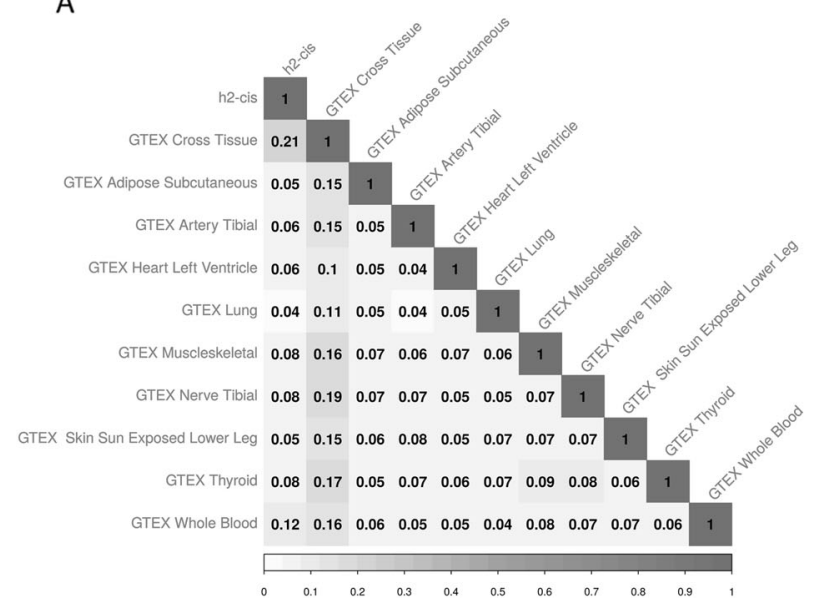

B

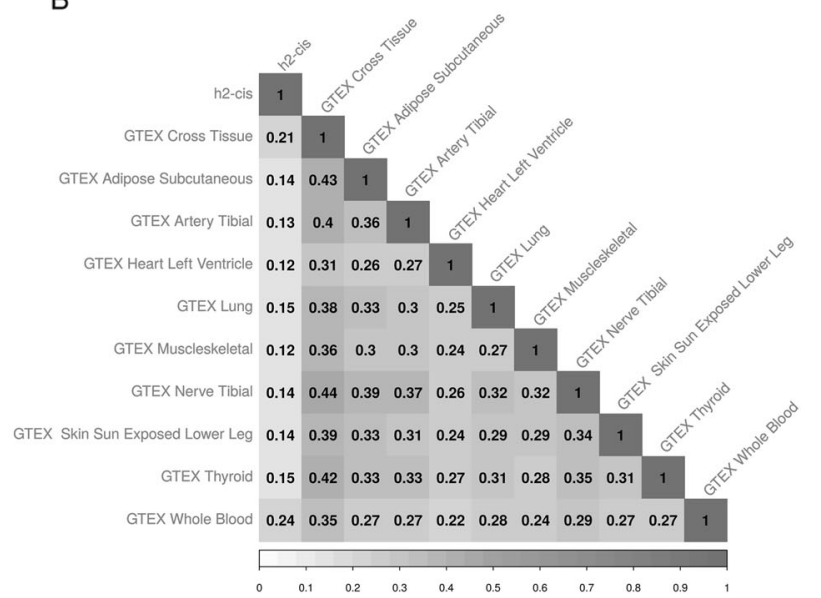

Fig. 2 Spearman correlations between $h_{\text {cis }}^{2}$ and estimates of GTEx per-tissue heritabilities [27]. a Tissue-specific heritabilities. b Tissue-wide heritabilities

\section{Pathway analyses}

To annotate gene expression heritability, we studied if certain gene pathways have higher average heritability than expected. In order to so we tested for enrichment of $h^{2}$ res and $h_{\text {cis }}^{2}$ in canonical gene pathways covering a broad range of biological pathways that are well curated (KEGG, REACTOME, BIOCARTA). The expression of genes identified in GWAS is likely to be under genetic control: to test if this is the case for gene expression in blood, we also tested if $h_{\text {res }}^{2}$ and $h_{\text {cis }}^{2}$ is enriched in genes identified in genome-wide association studies (GWAS) for immune diseases, mental and behavioral disorders, cardiovascular diseases, or cancer (extracted from the GWAS catalog [25]) to cover GWAS findings for a broad range of diseases. Enrichment analysis were performed before and after correcting $h^{2}$ for median gene expression per gene.

We observed significantly higher $h_{\text {res }}^{2}$ (false discovery rate (FDR) <0.05) in 343 canonical pathways (top hit: KEGG cytokine-cytokine interaction, $p=4.22 \times 10^{-15}$ ), and in genes identified in GWAS for immune diseases $\left(p=4.99 \times 10^{-13}\right)$, mental disorders $\left(p=1.79 \times 10^{-7}\right)$, cancer $\left(p=5.53 \times 10^{-5}\right)$ and cardiovascular diseases $\left(p=1.06 \times 10^{-9}\right)$. After correction for mean gene expression, $h_{\text {res }}^{2}$ was significantly higher in 125 canonical pathways (top hit: KEGG cytokine-cytokine interaction, $p=6.66 \times 10^{-15}$ ) and in genes identified in GWAS for immune diseases $\left(p=2.33 \times 10^{-10}\right)$, mental disorders $\left(p=3.63 \times 10^{-10}\right)$, cancer $\left(p=7.10 \times 10^{-6}\right)$ and cardiovascular diseases $\left(p=9.69 \times 10^{-10}\right)$.

We found significantly higher $h_{\text {cis }}^{2}(\mathrm{FDR}<0.05)$ in 6 canonical pathways (top hit: KEGG lysosome, $p=1.41 \times$ $\left.10^{-7}\right)$, and in genes identified in GWAS for immune diseases $\left(p=6.71 \times 10^{-4}\right)$, mental disorders $\left(p=7.13 \times 10^{-4}\right)$, cancer $\left(p=1.09 \times 10^{-7}\right)$ and cardiovascular diseases $(p=$ $\left.3.24 \times 10^{-5}\right)$. After correction for mean gene expression, $h^{2}$ res was significantly higher in 10 canonical pathways (top hit: KEGG lysosome, $p=5.79 \times 10^{-9}$ ) and in genes identified in GWAS for immune diseases $\left(p=9.13 \times 10^{-5}\right)$, for mental disorders $\left(p=2.36 \times 10^{-3}\right)$, cancer $\left(p=3.15 \times 10^{-7}\right)$ and cardiovascular diseases $\left(p=1.16 \times 10^{-5}\right)$ (see Table 2 and Supplementary Table 5).

\section{Gene expression $\boldsymbol{h}^{2}$ correlations}

In order to identify physiological gene properties that are correlated with gene expression heritability, we evaluated the correlation between heritability and gene expression level, gene length, GC content and several loss-of-function scores, see Table 3). We found a significant association of $h_{\text {res }}^{2}$ and $h_{\text {cis }}^{2}$ with median read count $\left(P=6.81 \times 10^{-276}\right.$, $P=2.15 \times 10^{-2}$, respectively) and GC content of a gene $\left(P<1.80 \times 10^{-115}, \quad P=4.02 \times 10^{-26}\right.$, respectively). After correcting for median read count, GC content was still significantly correlated with $h_{\text {res }}^{2}$ and $h^{2 \text { cis }}\left(\mathrm{P}=2.27 \times 10^{-3}\right.$, $P=6.11 \times 10^{-28}$, respectively). The length of a gene was significantly correlated with $h^{2}$ res, with longer genes having a slightly higher $h_{\text {res }}^{2}(P=4.94 \times 10-8)$. Gene length did not influence $h_{\text {cis }}^{2}(P=.50)$. A high intolerance to LoF or high probability of loss-of-function (pLI) did not significantly influence $h^{2}$ estimates.

\section{Relation of $\boldsymbol{h}^{2}$ cis with strength of cis-eQTLs}

Gene expression can have substantial $h^{2}$ not explained by eQTLs [6]. In order to study the overlap between heritability and eQTL results, we performed eQTL analysis in the same sample (see Methods) and found 5249 genes with a significant cis-eQTL ( $p$-value threshold $1.5 \times 10^{-7}$ for a FDR of 5\%). In addition, we found a significant association between $h_{\text {cis }}^{2}$ and the beta of the corresponding top cis- 
Table 2 Gene pathway enrichment of heritable genes in certain gene pathways (KEGG, REACTOME, BIOCARTA) and genes identified in GWAS for immune diseases, mental or behavioral disorders, cardiovascular diseases, or cancer (extracted from the GWAS catalog [25])
Table 3 Predictors of $h^{2}$

\begin{tabular}{|c|c|c|}
\hline & $\begin{array}{l}h_{\text {res }}^{2} \text { enrichment } \\
p \text {-value }\end{array}$ & $\begin{array}{l}h_{\text {cis }}^{2} \text { enrichment } \\
p \text {-value }\end{array}$ \\
\hline All immune-related GWAS genes & $4.99 \times 10^{-13}$ & $6.71 \times 10^{-4}$ \\
\hline All immune-related GWAS genes ${ }^{\mathrm{a}}$ & $2.33 \times 10^{-10}$ & $9.13 \times 10^{-5}$ \\
\hline All psychiatric-related GWAS genes & $1.79 \times 10^{-7}$ & $7.13 \times 10^{-4}$ \\
\hline All psychiatric-related GWAS genes ${ }^{\mathrm{a}}$ & $3.63 \times 10^{-10}$ & $2.36 \times 10^{-3}$ \\
\hline All cancer-related GWAS genes & $5.53 \times 10^{-5}$ & $1.09 \times 10^{-7}$ \\
\hline All cancer-related GWAS genes ${ }^{a}$ & $7.10 \times 10^{-6}$ & $3.15 \times 10^{-7}$ \\
\hline All cardiovascular GWAS genes & $1.06 \times 10^{-9}$ & $3.24 \times 10^{-5}$ \\
\hline All cardiovascular GWAS genes ${ }^{\mathrm{a}}$ & $9.69 \times 10^{-10}$ & $1.16 \times 10^{-5}$ \\
\hline KEGG_CYTOKINE_CYTOKINE_RECEPTOR_INTERACTION & $4.22 \times 10^{-15}$ & \\
\hline KEGG_CYTOKINE_CYTOKINE_RECEPTOR_INTERACTION ${ }^{\mathrm{a}}$ & $6.66 \times 10^{-15}$ & \\
\hline KEGG_LYSOSOME & & $1.41 \times 10^{-7}$ \\
\hline KEGG_LYSOSOME ${ }^{\mathrm{a}}$ & & $5.79 \times 10^{-9}$ \\
\hline
\end{tabular}

${ }^{\mathrm{a}} h^{2}$ corrected for median read count. See also Supplementary Table 5.

\begin{tabular}{|c|c|c|c|c|c|c|}
\hline \multirow[b]{2}{*}{ Predictor } & \multicolumn{3}{|l|}{$h_{\text {res }}^{2}$} & \multicolumn{3}{|l|}{$h_{\text {cis }}^{2}$} \\
\hline & Estimate & $P$-value & r-squared & Estimate & $P$-value & r-squared \\
\hline \multirow[t]{2}{*}{ Median Read count } & $5.21 \times 10^{-2}$ & $6.81 \times 10^{-276}$ & $1.16 \times 10^{-1}$ & $-3.76 \times 10^{-3}$ & $2.15 \times 10^{-2}$ & $8.16 \times 10^{-4}$ \\
\hline & NA & NA & NA & NA & NA & NA \\
\hline GC content & $3.10 \times 10^{-3}$ & $1.80 \times 10^{-115}$ & $4.81 \times 10^{-2}$ & $1.58 \times 10^{-3}$ & $4.02 \times 10^{-26}$ & $8.85 \times 10^{-3}$ \\
\hline GC content $\mathrm{a}^{\mathrm{a}}$ & $2.27 \times 10^{-3}$ & $1.07 \times 10^{-69}$ & $2.83 \times 10^{-2}$ & $1.64 \times 10^{-3}$ & $6.11 \times 10^{-28}$ & $9.85 \times 10^{-3}$ \\
\hline LOF score & $7.24 \times 10^{-3}$ & $8.16 \times 10^{-} 18$ & $3.14 \times 10^{-2}$ & $-3.19 \times 10^{-3}$ & $8.52 \times 10^{-5}$ & $2.55 \times 10^{-3}$ \\
\hline LOF score ${ }^{a}$ & $-1.65 \times 10^{-4}$ & $8.04 \times 10^{-1}$ & $3.95 \times 10^{-6}$ & $6.10 \times 10^{-4}$ & $4.08 \times 10^{-1}$ & $6.18 \times 10^{-5}$ \\
\hline pLI score & $3.54 \times 10^{-2}$ & $7.09 \times 10^{-19}$ & $2.47 \times 10^{-2}$ & $-2.37 \times 10^{-2}$ & $1.27 \times 10^{-9}$ & $7.41 \times 10^{-3}$ \\
\hline pLI score ${ }^{a}$ & $-1.73 \times 10^{-3}$ & $5.98 \times 10^{-1}$ & $1.50 \times 10^{-5}$ & $-3.00 \times 10^{-3}$ & $4.13 \times 10^{-1}$ & $1.29 \times 10^{-4}$ \\
\hline pNull & $-4.59 \times 10^{-2}$ & $2.86 \times 10^{-17}$ & $2.54 \times 10^{-2}$ & $1.72 \times 10^{-2}$ & $6.30 \times 10^{-4}$ & $1.90 \times 10^{-3}$ \\
\hline $\mathrm{pNull}^{\mathrm{a}}$ & $-4.31 \times 10^{-3}$ & $2.99 \times 10^{-1}$ & $7.50 \times 10^{-5}$ & $-3.54 \times 10^{-3}$ & $4.45 \times 10^{-1}$ & $7.75 \times 10^{-5}$ \\
\hline $\mathrm{pRec}$ & $-1.30 \times 10^{-2}$ & $3.25 \times 10^{-3}$ & $1.43 \times 10^{-3}$ & $1.58 \times 10^{-2}$ & $1.96 \times 10^{-4}$ & $3.34 \times 10^{-3}$ \\
\hline $\mathrm{pRec}^{\mathrm{a}}$ & $-1.64 \times 10^{-3}$ & $6.50 \times 10^{-1}$ & $5.74 \times 10^{-5}$ & $7.37 \times 10^{-3}$ & $6.62 \times 10^{-2}$ & $4.33 \times 10^{-4}$ \\
\hline Gene length & $1.80 \times 10^{-8}$ & $8.10 \times 10^{-2}$ & $2.12 \times 10^{-4}$ & $9.54 \times 10^{-9}$ & $3.86 \times 10^{-1}$ & $2.30 \times 10^{-4}$ \\
\hline Gene length $^{\mathrm{a}}$ & $4.94 \times 10^{-8}$ & $4.13 \times 10^{-7}$ & $2.26 \times 10^{-3}$ & $7.48 \times 10^{-9}$ & $4.96 \times 10^{-1}$ & $1.59 \times 10^{-4}$ \\
\hline
\end{tabular}

We tested whether $h^{2}$ could be predicted by gene expression level, gene length, GC content or several lossof-function scores obtained from Lek et al. [22]

$L O F$ loss-of-function, $P L I$ probability of loss-of-function, pNull completely tolerant of loss-of-function, $p R e c$ intolerant of two loss-of-function variants

${ }^{\mathrm{a}} h^{2}$ corrected for median read count
eQTLs $\left(\rho=0.7644, p<10^{-308}\right)$. We also tested the correlation between $h_{\text {cis }}^{2}$ and the presence of cis-eQTLs in results from Zhernakova et al. [16], who performed RNA-Seqbased eQTL analysis in an independent sample $(N=2116$ unrelated adults). There was a strong correlation between $h_{\text {cis }}^{2}$ and the $Z$-score of the strongest eQTL $\left(\rho=0.75, p<10^{-308}\right)$ (Supplementary Fig. 9).

\section{Relation of $\boldsymbol{h}^{2}$ res with strength of trans-eQTLs}

We performed eQTL analysis in the same sample for SNPs outside a cis-window of $250 \mathrm{~kb}$ around each gene, resulted in
2433 genes with a significant trans-eQTL ( $p$-value threshold $1.5 \times 10^{-7}$ for a FDR of $\left.5 \%\right)$. Estimates of $h_{\text {res }}^{2}$ correlated with the beta of the corresponding top trans-eQTLs $(\rho=.04$, $\left.p=1.89 \times 10^{-3}\right)$. The mean $p$-value of trans-eQTL of genes with $h_{\text {res }}^{2}>0.4$ was $5.51 \times 10^{-9}\left(\right.$ median $\left.=1.44 \times 10^{-9}\right)$.

\section{Comparing RNA-Seq and micro-arrays: relation of mean expression levels with heritability}

The difference in heritability estimates between RNAseq and array data results, especially with gene expression intensity as a factor, is a valuable comparison to distinguish noise or 


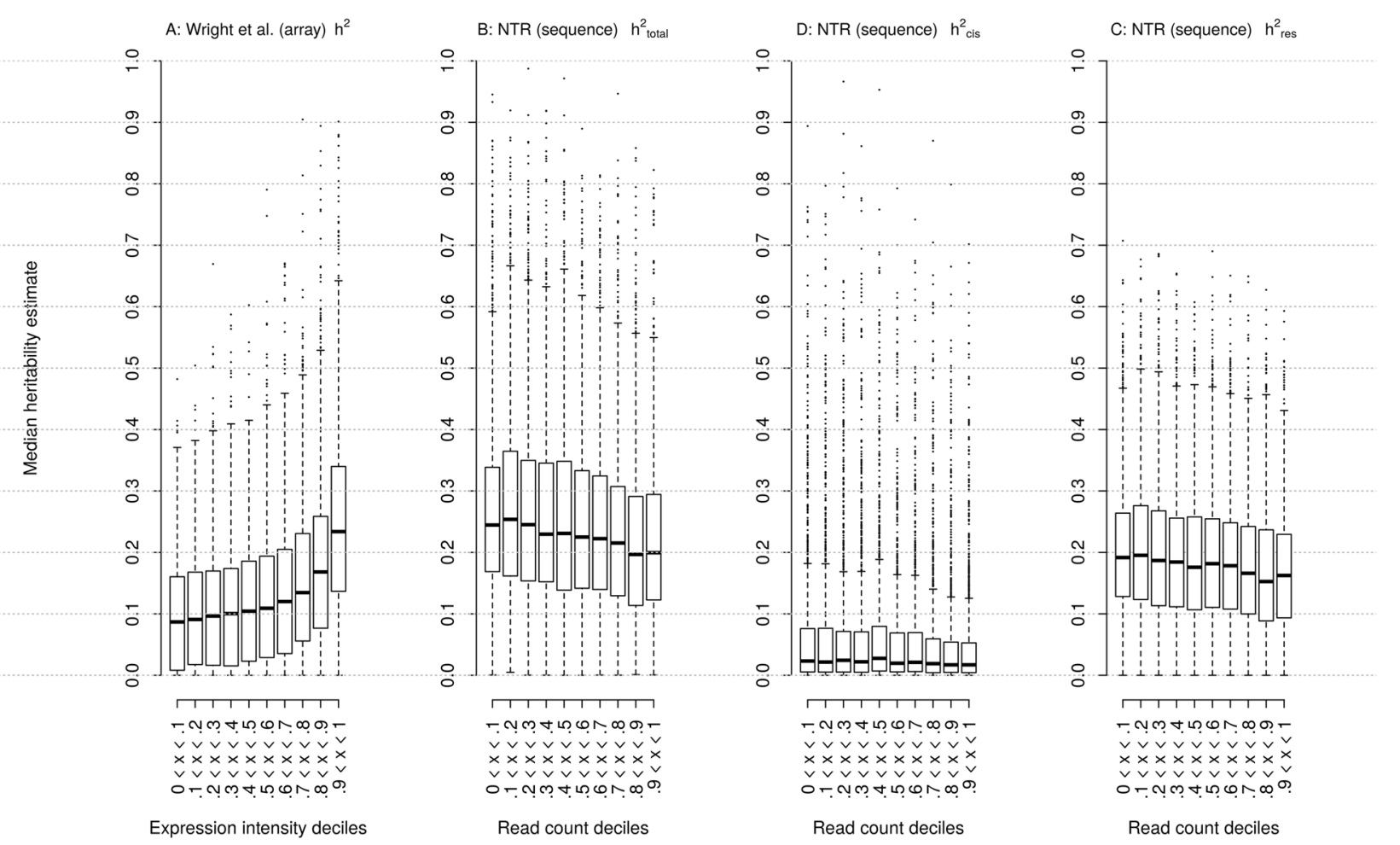

Fig. 3 Heritability estimates for genes divided into groups based on gene expression levels per decile. a Total heritability estimates from Wright et al. [7] b IBS-based $h_{\text {total }}^{2}$ estimates. c IBS-based $h_{\text {cis }}^{2}$ estimates. d IBS-based $h_{\text {res }}^{2}$ estimates

bias that is inherent to a specific technique. As RNAseq is a more expensive method, it is informative to know if and in which situations this technique offers advantages to answer a particular research question. From our original results on 52,844 genes, we selected the 12,070 genes that were measured both with Affymetrix expression arrays in the study by Wright et al. [7] and with RNA-Seq with read counts above zero in at least $85 \%$ of samples in each zygosity group. In contrast to previous analyses, we did not apply any additional filtering (i.e. minimum median read count per gene, protein coding genes only, minimum of SNPs per GRM to allow for an unbiased comparison. In the RNA-Seq data, the Spearman correlation of mean expression with $h_{\text {total }}^{2}$ was -0.13 after correcting for covariates (see Methods) $\left(p=7.49 \times 10^{-45}\right)$. In the array-based results from Wright et al. [7] the Spearman correlation between mean expression and $h^{2}$ was $0.28\left(p=2.08 \times 10^{-219}\right)$ after correcting for covariates.

Using this selection of 12,070 genes, mean $h_{\text {res }}^{2}, h_{\text {cis, }}^{2}$ $h_{\text {total }}^{2}$, and $h_{\text {total }}^{2}$ results from Wright et al. [7] were $0.19,0.06$, 0.25 , and 0.14 , respectively. When we partitioned mean expression levels into 10 deciles for both microarray and RNA-Seq data, we saw that $h_{\text {res }}^{2}$ estimates were also higher in RNA-Seq data for almost all deciles (see Fig. 3 and Supplementary Table 6), with the difference being largest in the lowest deciles $\left(p=1.06 \times 10^{-72}\right)$. In the highest decile of expression, the $h^{2}$ of array-based expression was higher $\left(p=2.00 \times 10^{-6}\right)$. This suggests that the resolution of RNASeq is better able to capture variation in low to moderately expressed genes. Genes measured by both array and RNASeq were mostly in the same or nearest decile (see Supplementary Fig. 10). Estimates of $h_{\text {cis }}^{2}$ showed a slight negative correlation with median expression level in the RNA-Seq data eQTLs $\left(\rho=-0.04, p<5.79 \times 10^{-5}\right)$.

\section{Discussion}

The present study estimated the $h^{2}$ of gene expression in RNA-Seq-based expression data by making use of the different genetic relatedness of mono- and dizygotic twins and an IBS approach. The mean of total gene expression $h^{2}$ (0.26) was substantially higher than found with the microarray-based study by Wright et al. with largely overlapping RNA samples. This was also the case with a direct comparison of 12,070 genes that were measured both with Affymetrix expression arrays in the study by Wright et al. [7] and with RNA-Seq, where mean RNA-Seq-based $h_{\text {total }}^{2}$ was 0.25 and mean microarray-based $h_{\text {total }}^{2}$ was $0.14\left(p<10^{-308}\right)$. Heritability estimates in RNA-Seq did not increase with gene expression level, as opposed to the results from microarray data. This suggests RNA-Seq measurements are less noisy, in particular in genes with low expression, as compared to microarrays measures. 
We estimated $h^{2}$ of gene expression at 0.20 for mean $h^{2}$ res, and at 0.06 for mean $h_{\text {cis }}^{2}$. This resulted in a relative contribution of $h_{\text {cis }}^{2}$ to $h_{\text {total }}^{2}$ of $20 \%$. This is in line with earlier findings by Wright et al. [7] (relative contribution of local IBS-driven $h^{2}$ of 23\%) and Lloyd-Jones et al. [6] (proportion of $h^{2}$ explained by cis-eQTL of 0.31). Since local variants in the cis-window of a gene only explain $20 \%$ on average of the total $h^{2}$, and the number of genes for which the majority of heritability stems from genetic relatedness in the cis-window is very low, our findings indicate that loci outside the cis-window of a gene or rare local variants explain a significant proportion of total $\mathrm{h}^{2}$ of gene expression. This is strengthened by our finding that the strength of a trans-eQTL is correlated with $h_{\text {res. }}^{2}$.

The $h_{\text {cis }}^{2}$ estimates correlated strongly with estimates from an independent sample $(\rho=0.81$, whole blood, $N=$ 922) [26], but much less so in the smaller GTEx dataset [27] ( $\rho=0.24$, whole blood, $N=449)$. This shows that reasonably large sample sizes are needed for accurate $h^{2}$ estimation. However, even with the small sample size used in GTEx we found significant correlations between $h_{\text {cis }}^{2}$ in whole blood and $h_{\text {cis }}^{2}$ in the other tissues, which is in line with the finding that cis-eQTLs are partially shared between tissues [29].

We found a significantly higher $h_{\text {res }}^{2}$ in 125 canonical pathways, with the strongest enrichment for genes in the KEGG cytokine-cytokine interaction pathway, and many other immune system pathways (including KEGG Innate Immune system $\left(p=8 \times 10^{-12}\right)$, REACTOME interferon signaling $\left(p=2 \times 10^{-8}\right)$ and KEGG natural killer cell cytotoxicity $\left.\left(p=2 \times 10^{-7}\right)\right)$ [30].

Both $h_{\text {cis }}^{2}$ and $h_{\text {res }}^{2}$ were higher in all genes identified in GWAS for immune diseases, mental disorders, and cardiovascular diseases, although interestingly the enrichment

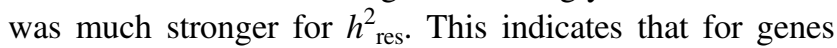
associated with a disease through GWAS, expression in blood is not only locally regulated but also enriched with genome-wide SNP signal as reflected in the high average $h_{\text {res }}^{2}$, suggesting that SNPs found in GWAS are influencing expression of genes outside the cis-window of the gene. A high intolerance to loss-of-function (LoF) or high probability of loss-of-function (pLI) did not significantly influence $h^{2}$ estimates, suggesting that increased mutational load in a gene increases the genetic variation as much as it influences variation in gene expression and therefore does not influence $h^{2}$.

The comparison of mean $h^{2}$ of genes across different RNA measurement techniques can be viewed as a proxy for the comparison of the measurement error between techniques. An increase in measurement error always implies a decrease in $h^{2}$ (because measurement error introduces random divergence within twin pairs). Consider for example the $h^{2}$ of probes conditioned on the median expression level.
When comparing the $h_{\text {res }}^{2}$ estimates obtained based on RNA-Seq with those obtained based on Affymetrix expression arrays [7], it becomes apparent that micro-arraybased estimates of gene expression heritability are associated with gene expression levels. The fact that differential measurement error conditional on expression level plays less of a role for RNA-Seq data ensures that variation across genes reflects biological signal. The slight negative correlation we found of $h_{\text {cis }}^{2}$ with median expression level in the RNA-Seq data eQTLs $\left(\rho=-0.04, p<5.79 \times 10^{-5}\right)$ is counterintuitive, difficult to interpret and presumably not meaningful.

If, or when, researchers eventually examine the $h^{2}$ of RNA expression levels in single cells, or nuclei, an inspection of the relationship between median expression levels and $h^{2}$ can be used to detect this source of differential measurement error.

In summary, this study shows possible advantages of $h_{\text {res-informed trans-eQTL discovery, reproducibility of }}^{2}$ $h_{\text {cis }}^{2}$, and the benefits of using RNA-Seq for estimating heritability of low-expressed genes.

Acknowledgements We very warmly thank all participants in the study. This study makes use of data in the Netherlands Twin Register with prof. D.I. Boomsma as principle investigator.

Funding This work was performed within the framework of the BBMRI - NL Consortium, a research infrastructure financed by the Dutch government (NWO, nos. 184.021.007 and 184.033.111). Genotyping was made possible by grants from NWO/SPI 56-464-14192, Genetic Association Information Network (GAIN) of the Foundation for the National Institutes of Health, Rutgers University Cell and DNA Repository (NIMH U24 MH068457-06), the Avera Institute, Sioux Falls (USA) and the National Institutes of Health (NIH R01 HD042157-01A1, MH081802, Grand Opportunity grants 1RC2 MH089951 and 1RC2 MH089995) and European Research Council (ERC-230374). DIB acknowledges her KNAW Academy Professor Award (PAH/6635).

${ }^{12}$ Molecular Epidemiology Section, Department of Medical Statistics and Bioinformatics, Leiden University Medical Center, Leiden, The Netherlands; ${ }^{13}$ Department of Human Genetics, Leiden University Medical Center, Leiden, The Netherlands; ${ }^{14}$ Department of Internal Medicine, ErasmusMC, Rotterdam, The Netherlands; ${ }^{15}$ Department of Genetic Epidemiology, ErasmusMC, Rotterdam, The Netherlands; ${ }^{16}$ Department of Psychiatry, VU University Medical Center, Neuroscience Campus Amsterdam, Amsterdam, The Netherlands; ${ }^{17}$ Department of Genetics, University of Groningen, University Medical Centre Groningen, Groningen, The Netherlands; ${ }^{18}$ Department of Biological Psychology, VU University Amsterdam, Neuroscience Campus Amsterdam, Amsterdam, The Netherlands; ${ }^{19}$ Department of Internal Medicine and School for Cardiovascular Diseases (CARIM), Maastricht University Medical Center, Maastricht, The Netherlands;

${ }^{20}$ Department of Gerontology and Geriatrics, eiden University Medical Center, Leiden, The Netherlands; ${ }^{21}$ Department of Neurology, Brain Center Rudolf Magnus, University Medical Center Utrecht, Utrecht, The Netherlands; ${ }^{22}$ Department of Epidemiology, ErasmusMC, Rotterdam, The Netherlands; ${ }^{23}$ Sequence Analysis Support Core, Leiden University Medical Center, Leiden, The Netherlands; ${ }^{24}$ SURFsara, Amsterdam, The Netherlands; ${ }^{25}$ Genomics Coordination 
Center, University Medical Center Groningen, University of Groningen, Groningen, The Netherlands; ${ }^{26}$ Medical Statistics Section, Department of Medical Statistics and Bioinformatics, Leiden University Medical Center, Leiden, The Netherlands

BIOS Consortium Bastiaan T. Heijmans ${ }^{12}$, Peter A. C. 't Hoen ${ }^{13}$, Joyce van Meurs ${ }^{14}$, Aaron Isaacs ${ }^{15}$, Rick Jansen ${ }^{16}$, Lude Franke ${ }^{17}$, Dorret I. Boomsma $^{18}$, René Pool ${ }^{18}$, Jenny van Dongen ${ }^{18}$, Jouke J. Hottenga ${ }^{18}$, Marleen M. J. van Greevenbroek ${ }^{19}$, Coen D. A. Stehouwer ${ }^{19}$, Carla J. H. van der Kallen ${ }^{19}$, Casper G. Schalkwijk ${ }^{19}$, Cisca Wijmenga ${ }^{17}$, Lude Franke $^{17}$, Sasha Zhernakova ${ }^{17}$, Ettje F. Tigchelaar ${ }^{17}$, P. Eline Slagboom $^{12}$, Marian Beekman ${ }^{12}$, Joris Deelen ${ }^{12}$, Diana van Heemst ${ }^{20}$, Jan H. Veldink ${ }^{21}$, Leonard H. van den Berg ${ }^{21}$, Cornelia M. van Duijn ${ }^{15}$, Bert A. Hofman ${ }^{22}$, Aaron Isaacs ${ }^{15}$, André G. Uitterlinden ${ }^{14}$, Joyce van Meurs $^{14}$, P. Mila Jhamai ${ }^{14}$, Michael Verbiest ${ }^{14}$, H. Eka D. Suchiman ${ }^{12}$, Marijn Verkerk ${ }^{14}$, Ruud van der Breggen ${ }^{12}$, Jeroen van Rooij ${ }^{14}$, Nico Lakenberg $^{12}$, Hailiang $\mathrm{Mei}^{23}$, Maarten van Iterson ${ }^{12}$, Michiel van Galen $^{13}$, Jan Bot ${ }^{24}$, Dasha V. Zhernakova ${ }^{17}$, Rick Jansen ${ }^{16}$, Peter van't Hof $^{23}$, Patrick Deelen ${ }^{17}$, Irene Nooren ${ }^{24}$, Peter A. C. 't Hoen ${ }^{13}$, Bastiaan T. Heijmans ${ }^{12}$, Matthijs Moed ${ }^{12}$, Lude Franke ${ }^{17}$, Martijn Vermaat $^{14}$, Dasha V. Zhernakova ${ }^{17}$, René Luijk ${ }^{12}$, Marc Jan Bonder ${ }^{17}$, Maarten van Iterson ${ }^{12}$, Patrick Deelen ${ }^{17}$, Freerk van Dijk ${ }^{25}$, Michiel van Galen ${ }^{13}$, Wibowo Arindrarto ${ }^{23}$, Szymon M. Kielbasa ${ }^{26}$, Morris A. Swertz $^{25}$, Erik. W van Zwet ${ }^{26}$, Rick Jansen ${ }^{16}$, Peter-Bram 't Hoen ${ }^{13}$, Bastiaan T. Heijmans ${ }^{12}$

\section{Compliance with ethical standards}

Conflict of interest The authors declare that they have no conflict of interest.

Ethical approval The NTR study was approved by the Central Ethics Committee on Research Involving Human Subjects of the VU University Medical Center, Amsterdam (institutional review board [IRB] number IRB-2991 under Federal wide Assurance 3703; IRB/institute code NTR 03-180). All participants provided written informed consent.

Publisher's note Springer Nature remains neutral with regard to jurisdictional claims in published maps and institutional affiliations.

\section{References}

1. Leek JT, Storey JD. Capturing heterogeneity in gene expression studies by surrogate variable analysis. PLoS Genet. 2007;3:1724-35.

2. Bryois J, Buil A, Ferreira PG, Panousis NI, Brown AA, Viñuela A, et al. Time-dependent genetic effects on gene expression implicate aging processes. Genome Res. 2017;27:545-52.

3. Jansen R, Batista S, Brooks AI, Tischfield JA, Willemsen G, van Grootheest G, et al. Sex differences in the human peripheral blood transcriptome. BMC Genomics. 2014;15:33.

4. Meder B, Backes C, Haas J, Leidinger P, Stähler C, Großmann T, et al. Influence of the confounding factors age and sex on microRNA profiles from peripheral blood. Clin Chem. 2014;60:1200-8.

5. Tower J. Sex-specific gene expression and life span regulation. Trends Endocrinol Metab. 2017;28:735-47.

6. Lloyd-Jones LR, Holloway A, McRae A, Yang J, Small K, Zhao $\mathrm{J}$, et al. The genetic architecture of gene expression in peripheral blood. Am J Hum Genet. 2017;100:228-37.

7. Wright FA, Sullivan PF, Brooks AI, Zou F, Sun W, Xia K, et al. Heritability and genomics of gene expression in peripheral blood. Nat Genet. 2014;46:430-7.
8. Vinuela A, Brown AA, Buil A, Tsai PC, Davies MN, Bell JT, et al. Age-dependent changes in mean and variance of gene expression across tissues in a twin cohort. Hum Mol Genet. 2018;27:732-41.

9. Vink JM, Jansen R, Brooks A, Willemsen G, van Grootheest G, de Geus E, et al. Differential gene expression patterns between smokers and non-smokers: cause or consequence? Addiction Biol. 2017;22:550-60.

10. Willemsen G, de Geus EJ, Bartels M, van Beijsterveldt CE, Brooks AI. Estourgie-van Burg GF et al: The Netherlands Twin Register biobank: a resource for genetic epidemiological studies. Twin Res Hum Genet. 2010;13:231-45.

11. Willemsen G, Vink JM, Abdellaoui A, den Braber A, van Beek $\mathrm{JH}$, Draisma $\mathrm{HH}$, et al. The adult netherlands twin register: twenty-five years of survey and biological data collection. Twin Res Hum Genet. 2013;16:271-81.

12. Boomsma D, Busjahn A, Peltonen L. Classical twin studies and beyond. Nat Rev Genet. 2002;3:872-82.

13. Yang J, Lee SH, Goddard ME, Visscher PM. GCTA: a tool for genome-wide complex trait analysis. Am J Hum Genet. 2011;88:76-82.

14. Willemsen G, Vink JM, Abdellaoui A, den Braber A, van Beek JH, Draisma HH, et al. The Adult Netherlands Twin Register: twenty-five years of survey and biological data collection. Twin Res Hum Genet. 2013;16:271-81.

15. Genome of the Netherlands C. Whole-genome sequence variation, population structure and demographic history of the Dutch population. Nat Genet. 2014;46:818-25.

16. Zhernakova DV, Deelen P, Vermaat M, van Iterson M, van Galen $\mathrm{M}$, Arindrarto $\mathrm{W}$, et al. Identification of context-dependent expression quantitative trait loci in whole blood. Nat Genet. 2017;49:139-45.

17. Boomsma DI, Wijmenga C, Slagboom EP, Swertz MA, Karssen LC, Abdellaoui A, et al. The Genome of the Netherlands: design, and project goals. Eur J Hum Genet. 2014;22:221-7.

18. R Development Core Team. R: a language and environment for statistical computing. Vienna: R Foundation for Statistical Computing; 2014.

19. Neale MC, Hunter MD, Pritikin JN, Zahery M, Brick TR, Kirkpatrick RM, et al. OpenMx 2.0: extended structural equation and statistical modeling. Psychometrika. 2016;81:535-49.

20. Zaitlen N, Kraft P, Patterson N, Bogdan P, Gaurav, Samuela P, et al. Using extended genealogy to estimate components of heritability for 23 quantitative and dichotomous traits. PLoS Genet. 2013;9:e1003520.

21. Visscher PM, Hemani G, Vinkhuyzen AA, Chen GB, Lee SH, Wray NR, et al. Statistical power to detect genetic (co) variance of complex traits using SNP data in unrelated samples. PLoS Genet. 2014;10:e1004269.

22. Lek M, Karczewski KJ, Minikel EV, Samocha KE, Banks E, Fennell T, et al. Analysis of protein-coding genetic variation in 60,706 humans. Nature. 2016;536:285-91.

23. Smedley D, Haider S, Durinck S, Pandini L, Provero P, Allen J, et al. The BioMart community portal: an innovative alternative to large, centralized data repositories. Nucleic acids Res. 2015;43: W589-W598.

24. Kinsella RJ, Kähäri A, Haider S, Zamora J, Proctor G, Spudich G et al: Ensembl BioMarts: a hub for data retrieval across taxonomic space. Database 2011;2011:bar030.

25. MacArthur J, Bowler E, Cerezo M, Gil L, Hall P, Hastings E, et al. The new NHGRI-EBI Catalog of published genome-wide association studies (GWAS Catalog). Nucleic Acids Res. 2017;45: D896-901.

26. Battle A, Mostafavi S, Zhu X, Patash JB, Weissman MM, McCormick $\mathrm{C}$, et al. Characterizing the genetic basis of 
transcriptome diversity through RNA-sequencing of 922 individuals. Genome Res. 2014;24:14-24.

27. Consortium GT, Laboratory DA, Coordinating Center -Analysis Working G, Enhancing GTEx (eGTEx) groups, NIH Common Fund, NIH/NCI, et al. Genetic effects on gene expression across human tissues. Nature. 2017;550:204-13.

28. Franić S, Dolan CV, Borsboom D, Boomsma DI. Structural equation modeling in genetics. In: Hoyle RH (ed) Handbook of structural equation modeling. New York: Guilford Press; 2012. pp 617-35.

29. Wheeler HE, Shah KP, Brenner J, Garcia T, Aquino-Michaels K, GTEX Consortium et al. Survey of the heritability and sparse architecture of gene expression traits across human tissues. PLoS Genet. 2016;12:e1006423.

30. Kanehisa M, Furumichi M, Tanabe M, Sato Y, Morishima K. KEGG: new perspectives on genomes, pathways, diseases and drugs. Nucleic Acids Res. 2016;45:D353-61.

\section{Affiliations}

\section{Klaasjan G. Ouwens $\mathbb{D}^{1} \cdot$ Rick Jansen $\mathbb{D}^{2} \cdot$ Michel G. Nivard $\mathbb{D}^{1} \cdot$ Jenny van Dongen ${ }^{1} \cdot$ Maia J. Frieser ${ }^{3,4}$.

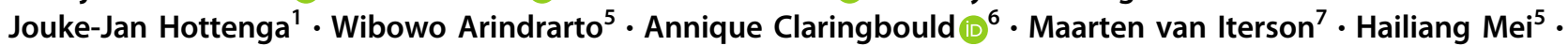 Lude Franke $\mathbb{1}^{6} \cdot$ Bastiaan T. Heijmans ${ }^{7} \cdot$ Peter A. C. 't Hoen $\mathbb{D}^{8,9} \cdot$ Joyce van Meurs ${ }^{10}$. Andrew I. Brooks ${ }^{11}$. BIOS Consortium • Brenda W. J. H. Penninx ${ }^{2} \cdot$ Dorret I. Boomsma ${ }^{1}{ }^{1}$}

1 Department of Biological Psychology, Amsterdam Public Health research institute, Vrije Universiteit Amsterdam, Amsterdam, The Netherlands

2 Department of Psychiatry, Amsterdam Public Health and Amsterdam Neuroscience, Amsterdam UMC, Vrije Universiteit, Amsterdam, The Netherlands

3 Department of Psychology and Neuroscience, University of Colorado Boulder, Boulder, USA

4 Institute for Behavioral Genetics, University of Colorado Boulder, Boulder, USA

5 Sequencing Analysis Support Core, Department of Biomedical Data Sciences, Leiden University Medical Center, Leiden, The Netherlands

6 Department of Genetics, University of Groningen, University
Medical Centre Groningen, Groningen, The Netherlands

7 Molecular Epidemiology, Department of Biomedical Data Sciences, Leiden University Medical Center, Leiden, The Netherlands

8 Department of Human Genetics, Leiden University Medical Center, Leiden, The Netherlands

9 Center for Molecular and Biomolecular Informatics, Radboud Institute for Molecular Life Sciences, Radboud University Medical Center Nijmegen, Nijmegen, the Netherlands

10 Department of Internal Medicine, ErasmusMC, Rotterdam, The Netherlands

11 Department of Genetics and the Human Genetics Institute, RUCDR Infinite Biologics, Rutgers University, New Brunswick, NJ, USA 